\title{
TCM Genomics - A Key Factor to Modernization of Traditional Chinese Medicine
}

\author{
Ming Yuan, Rufeng Wang*, Xiuwen Wu, Yannan An and Zhenxiao Sun* \\ School of Chinese Pharmaceutical Sciences, Beijing University of Chinese Medicine, Beijing 100102, China
}

Traditional Chinese medicine (TCM) has been proved to be effective during thousands of years of practice. It has formed an entire medicinal theoretical system. The unique effect of traditional Chinese medicine on many diseases has been widely recognized and has made great contribution to Chinese nation in the history. However, due to historical limitations and the great influx of Western medicine, its theoretical basis is difficult to be recognized by the modern society, especially the international community. Therefore, the modernization of Chinese medicine is imperative. Achieving the modernization of TCM needs to combine the rapid development of modern science and technology, especially biotechnology and genomic technology, to form a modern scientific explanation on the theory of traditional Chinese medicine [1]. Based on this theory, Chinese medicine combining with molecular biology and genomics established TCM Genomics.

TCM Genomics is based on molecular biology and traditional pharmacy. With tropism of taste and prescription compatible theory, it looks for target on which Chinese medicine acts and the law of TCM integral role [2]. In fact, it investigates the mechanisms and principles of TCM at genetic level and then pushes the traditional Chinese medicine to modernization.

The core of TCM Genomics is to study the effect of TCM on gene expression, especially disease-related gene expression. Specifically, it is a study on the relationship of medicinal properties, functions, indications and effects on gene expression. Using modern genomics theory, especially functional genomics and morbid genomics, we can illustrate traditional Chinese medicine theory and mechanism. In response to this core content, the first we should do is to look for some feasible techniques for the above content, such as biochip, genomics, proteomics and other technologies.

Chinese herbal medicine more often than not has complex compositions. It has formed its own unique genome overall structure in the process of its evolution. The essence of Chinese herbal medicine effect on human body seems like the relationship of Chinese herbal medicine genome and the human genome. Tropism of taste theory of TCM illustrates that material acts on genome targets of certain organs directly or indirectly [3].

From the perspective of genomics, molecular biology has become a powerful tool for identification, active ingredients acquiring, resource protection and pharmacological research of TCM [4]. Of course, it brings opportunities for traditional Chinese medicine research. As a bridge between genomics and Chinese medicine research, gene chip technology has characteristics of high-throughput, multi-factor, large-scale, miniaturization, sensitivity and celerity $[5,6]$. Gene chip technology has opened up a new field in drug target exploration, drug screening, pharmacology, toxicology, prescription research [58] of TCM and is now widely used in the modernization research of traditional Chinese medicine.

The fundamental point of TCM Genomics is to explain the genome principle of prescriptions, discover the entire mechanism through which TCM acts on human genome at the molecular level. Then, it makes prescriptions precision and simple, pushes TCM to the molecular level or finds the gene for active ingredients.
The modernization of basic theory is the key issue of the TCM modernization. TCM Genomics explores theoretical basis and treatment mechanism of TCM at the gene transcription and expression level. It is not only a summary of long-standing mechanism of TCM and the research results of material basis, but also provides a new mentality and orientation for modernization research of TCM theoretical basis.

Modernization of TCM is an inevitable trend of traditional Chinese medicine industry in the $21^{\text {st }}$ century. As emerging technologies in the field of life sciences, TCM Genomics explains the essence of substance in the micro-level and has become a powerful means to achieve the modernization of TCM. TCM Genomics combines TCM and genomics, diagnoses and treats disease, as well as improves human health authentically at the molecular level. Only by establishing TCM genomics, the advantages of TCM can be displayed and traditional Chinese medicine can really go to the world to serve the people all over the world.

\section{Acknowledgement}

This work was supported by the Free Topic Project of Beijing University of Chinese Medicine (No. 532/0100602099).

\section{References}

1. Wang SQ (2001) TCM Genomics and TCM Chemics. Capital Medicine 8: 1920.

2. Liu JQ (2009) On prescriptions genomics. Liaoning Zhong yi za zhi 36: 926.

3. Liu JQ (2004) Modernization of traditional Chinese medicine and establishment of TCM genomics. Chinese Archives of Traditional Chinese Medicine 22.

4. Mo XM (2006) Discussion for the application of molecular biology in the research of Chinese medicine. Journal of Modern Food and Pharmaceuticals 16: 75-77.

5. Zeng MG, Ji S (2005) Functional genomics and biological chip technology in the application of the modernization of traditional Chinese medicine. Fujian yi xue yuan xue bao 10: 98-101.

6. Jing ZW, Wang Z, Gao SH, Wang YY (2007) Microarray and research of Chinese medications: pharmcogenomics of Chinese medications. Zhongguo Zhong Yao Za Zhi 32: 289-292.

7. Sun DL, Shu QJ (2002) The meaning of gene expression profile in the study of traditional Chinese medicine. Zhong yi yao xin xi za zhi 9: 7-9.

8. Deng T, Ning ZQ, Zhou, Cheng J (2002) Application of biochip technology in drug discovery and development. Chinese Journal of New Drugs 11: 23-31.

*Corresponding authors: Rufeng Wang, School of Chinese Pharmaceutical Sciences, Beijing University of Chinese Medicine, Beijing 100102, China, Tel: + 86-10-84738646; E-mail: wangrufeng@tsinghua.org.cn

Zhenxiao Sun, School of Chinese Pharmaceutical Sciences, Beijing University of Chinese Medicine, Beijing 100102, China, Tel: + 86-10-84738646; E-mail: sunzxcn@hotmail.com

Received March 05, 2012; Accepted March 07, 2012; Published March 10, 2012

Citation: Yuan M, Wang R, Wu X, An Y, Sun Z (2012) TCM Genomics - A Key Factor to Modernization of Traditional Chinese Medicine. Single Cell Biol 1:e102. doi:10.4172/2168-9431.1000e102

Copyright: () 2012 Yuan M, et al. This is an open-access article distributed under the terms of the Creative Commons Attribution License, which permits unrestricted use, distribution, and reproduction in any medium, provided the original author and source are credited. 